\title{
Electrochemical Flow Injection Determination of Ascorbic Acid in Fruit Samples Employing a Graphene-Polyaniline Electrode
}

\author{
Poachanee Norfun, Ornanong Arqueropanyo, Saisunee Liawruangrath, and Kontad Ounnunkad
}

\begin{abstract}
A simple flow injection analysis (FIA) equipped with an electrochemical graphene (GP)-polyaniline (PANI) nanocomposite sensor as a detector has been investigated for determination of ascorbic acid (AA) in fruit samples. Northern Thai fruits (longans and lychees) harvested in Chiang Mai and Lamphun districts were selected for this study. The GP-PANI nanocomposite was interfacially polymerised and characterised by cyclic voltammetry. Observable electro-oxidation response of AA over the nanocomposite electrode with high peak current at a low potential was found. Optimisations of the experimental conditions were carried out which were found to be $0.1 \mathrm{M}$ phosphate buffer, pH 7, $1.5 \mathrm{~mL} \cdot \mathrm{min}^{-1}$ flow rate, $100 \mu \mathrm{L}$ sample injection volume, respectively. The good linear range of calibration curve, low limit of detection, and high sensitivity for the proposed method were found to be $0.05-0.50 \mathrm{mM}, 0.027 \mathrm{mM}$, and $4.5754 \mathrm{~mA} . \mathrm{mM}^{-1}$, respectively.
\end{abstract}

Index Terms-Graphene, polyaniline, amperometry, flow injection, ascorbic acid.

\section{INTRODUCTION}

A water-soluble vitamin, namely vitamin $\mathrm{C}$ or ascorbic acid (AA) could be observed in various biological systems and foods such as fresh vegetables and fruits. AA shows an important in iron absorption, collagen biosynthesis, and activation of immune response and assists in osteogenesis and wound healing. Interestingly, it actually acts as a powerful antioxidant that lowers free-radical induced damages [1], [2]. Nevertheless, gastric irritation due to an AA excess can generate the metabolic product of AA, causing renal problems [3]. Many analytical techniques have been proposed for the estimation of AA content such as chromatography [4], fluorimetry [5], spectrophotometry [6], redox titration [7], and electrochemical method [8]. Among these methods, the electrochemical method presents some advantages such as high sensitivity and ability to assay the electroactive species in coloured samples. However, the above batch-wise methods are time-consuming due to sample pre-treatments. They also consume large amounts of samples, expensive and/or toxic reagents and solvents. Therefore, the more rapid and greener analyses have been developed with miniaturisation. Flow injection analysis (FIA) seems promising to overcome such problems. The most simple and cost-effective flow injection

Manuscript received April 19, 2015; revised June 17, 2015.

The authors are with the Center of Excellence for Innovation in Chemistry (PERCH-CIC), Department of Chemistry, Faculty of Science, Chiang Mai University, Chiang Mai 50200, Thailand (e-mail: view-wow@ hotmail.com, orn.arquero@gmail.com, scislwrn@gmail.com, suriyacmu@yahoo.com).
(FI) manifold could be operated with the electrochemical detection.

Some chemically modified electrodes have been widely applied to decrease the oxidation overpotential and the detection of AA at the electrode surface/solution interface [1], [8], [9]. However, the electrodes usually lost their electroactivity easily by the unstable immobilisation of modifiers, resulted in unreliable determinations, thus causing inaccuracy in the measurement. In contrast, the modifications with electroactive polymers or nanocarbons reveal distinct advantages for three-dimensional mediator distribution, good electrode stability, and broad potential windows [9], [10]. Recently, polyaniline (PANI) has become an attractive electrode material in the field of electrochemical sensors and biosensors [11], [12]. In addition, incorporation of graphene (GP) into PANIs can provide good sensitivity and selectivity in the devices. This present work aims to investigate the FI-amperometric determination of AA towards direct oxidation of AA at the GP-PANI nanocomposite electrode. The developed method in the study was applied to assess the AA content in the most famous Northern Thai fruits (longans and lychees).

\section{PROCEDURE}

\section{A. Chemicals}

A $0.1 \mathrm{M}$ phosphate buffer ( $\mathrm{pH} 7.0), 0.1 \mathrm{M}$ hydrochloric acid $(\mathrm{HCl})$ and deionised water were used in this work Standard solutions of AA were freshly prepared in phosphate buffer before measurement.

\section{B. Sample Preparation}

Longans (Edor, Sri Choompoo and Haew) and lychees (Hong-Huay, O-hia and Chakrapad) used in this work were purchased from orchards in Chiang Mai and Lumphun districts, Thailand. The extraction was prepared as described by Leong and Shui with slightly modified [13]. Briefly, $50.0 \mathrm{~g}$ of edible tissue of each sample was mixed with $25.0 \mathrm{~mL}$ of $1: 1$ mixture of phosphate buffer and ethanol in a vortex. The solution was centrifuged at $2,500 \mathrm{rpm}$ for $10 \mathrm{~min}$ at a room temperature. The supernatant was filtered through a $0.45 \mu \mathrm{m}$ nylon membrane and used directly for determination of the AA content.

\section{Methods}

A glassy carbon electrode (GCE, $3.0 \mathrm{~mm}$ in diameter) employed as a working electrode was sequentially polished with $0.5,0.3$, and $0.1 \mu \mathrm{m} \mathrm{Al}_{2} \mathrm{O}_{3}$ slurries on a fine emery-paper. 
The electrode was sonicated in deionised water and then in ethanol for $1 \mathrm{~min}$ each and dried in air before use. For modification of GCE, $2.0 \mathrm{mg}$ interfacially polymerised GP-PANI was dispersed in $1.0 \mathrm{~mL}$ water under an ultrasonic wave for 15 mins. Then, $4.0 \mu \mathrm{L}$ of the dispersion was drop-coated onto the cleaned GCE surface and dried under light from desk lamp. Finally, the electrode was covered with $10.0 \mu \mathrm{L}$ of $1 \%$ Nafion $^{\circledR}$ solution and dried at room temperature. Cyclic voltammetric $(\mathrm{CV})$ characterisation was performed in order to evaluate the electrochemical behaviour of the nanocomposite sitting on GCE in $1.0 \mathrm{M} \mathrm{HCl}$ solution. The nanocomposite was prepared using $10 \mathrm{~mL}$ of $1.0 \mathrm{M} \mathrm{HCl}$ containing $0.18 \mathrm{~g}$ ammonium persulfate and $10 \mathrm{~mL}$ of chloroform $\left(\mathrm{CHCl}_{3}\right)$ containing $0.30 \mathrm{~mL}$ aniline and $\mathrm{GP}$ at concentration of $0.3 \mathrm{mg} \cdot \mathrm{mL}^{-1}$.

The proposed FI manifold for AA determination is shown in Fig. 1. The system consisted of a peristaltic pump (EYELA, Tokyo Rikakikal, Japan) with Tygon tubing (0.84 mm i.d. and $1.24 \mathrm{~mm}$ o.d., Cole-parmer, USA), a PTFE tubing $(0.84 \mathrm{~mm}$ i.d., $100 \mathrm{~cm}$ long, Cole-parmer, USA) mixing coil used as reactor, and the electrochemical flow-through cell constructed with the GP-PANI working electrode, an $\mathrm{Ag} / \mathrm{AgCl}$ reference electrode and a Pt counter electrode. The electrochemical measurements were performed by the eDAQ potentiostat (eDAQ, AUS) coupled with an eDAQ e-corder 410. Amperometric detection controlled by the eDAQ chart software at the cell was monitored for a flow of $0.1 \mathrm{M}$ phosphate buffer ( $\mathrm{pH} 7$ ) stream having the total flow rate of $3.0 \mathrm{mLmin}^{-1}$. A $125 \mu \mathrm{L}$ AA solution was injected into the stream via an injection valve. Then the solution was passed through in the electrochemical flow-through cell where AA was oxidised to its oxidised form at a working electrode with a constant applying potential (300 mV versus $\mathrm{Ag} / \mathrm{AgCl}(3.0 \mathrm{M}$ $\mathrm{NaCl})$ ). The current change is continuously recorded. Changes in amperometric signals were plotted against various concentrations of AA to give the calibration curve.

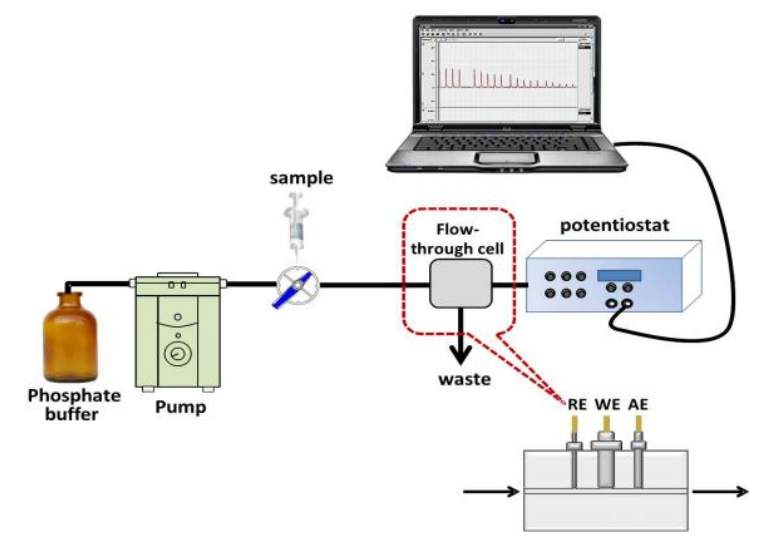

Fig. 1. Instrument setup for determination of AA using a GP-PANI nanocomposite electrode (WE).

\section{RESULT AND DISCUSSION}

Fig. 2 shows the cyclic voltammograms (CVs) of the GP-PANI-modified GCE obtained in $1.0 \mathrm{M} \mathrm{HCl}$ at different scan rates. Both of the oxidation and reduction peak currents (Fig. 2(a)) increased with the increasing scan rates. As shown in Fig. 2(b), cathodic and anodic peak currents $\left(\mathrm{mA} . \mathrm{cm}^{-2}\right)$ vary linearly with the scan rate $\left(\mathrm{mV}^{-1} \mathrm{~s}^{-1}\right)$ within the scan range of 10-300 mV.s ${ }^{-1}$. Linear regression analysis gave $R^{2}$ value of 0.9928 and 0.9967 . At low scan rates, CVs revealed the characteristic of electrochemical behaviour of PANI. Three redox couple peaks corresponded to the redox transition of PANI between the leucoemeraldine base and emeraldine salt, oxidation/reduction of head to tail dimer, and the transition of PANI between emeraldine salt and pernigraniline base [14]. This implies that GP-PANI nanocomposite shows good electroactivity.
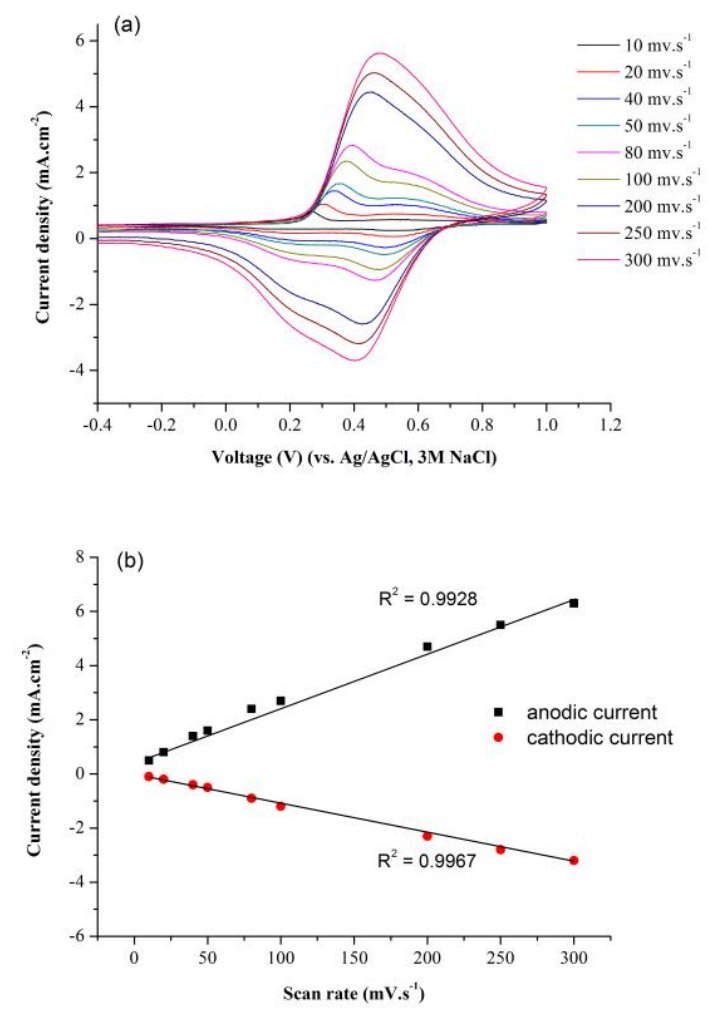

Fig. 2. (a) CVs of GP-PANI-modified GCE in $1.0 \mathrm{M} \mathrm{HCl}$ at different scan rates and (b) plots of anodic and cathodic currents versus scan rate.

Fig. 3(a) shows CVs of bare GCE and GCEs modified with different prepared nanomaterials in $1.0 \mathrm{M} \mathrm{HCl}$. Observable low currents of the CVs were found at bare and GP-modified GCEs. On the other hand, the GCEs modified PANI presented high currents due to the redox reactions of the PANI in pure PANI and PANI composite. It was found that addition of GP increased the peak currents of PANI and lowered the oxidation potential of the polymer. Furthermore, Fig. 3(b) showed that the CVs of the both electrodes in $1.0 \mathrm{mM} \mathrm{AA}$ containing $0.1 \mathrm{M}$ phosphate buffer $(\mathrm{pH} 7)$ at a scan rate of 100 $\mathrm{mV} . \mathrm{s}^{-1}$ exhibited good electrochemical oxidation of AA as compared to bare and GP-modified GCEs. The lowest oxidation potential at $300 \mathrm{mV}$ and highest current from AA oxidation were observed for the composite-modified GCE, suggesting highest electrode reactivity.

The FI parameters for the determination of AA were optimised by studying the chemical and physical factors that influenced the sensitivity and characteristic flow-injection parameters. Concentration of phosphate buffer, $\mathrm{pH}$ value of buffer solution, flow rates and sample volume injected were optimised, in an effort to obtain maximum amperometric response due to oxidation of $\mathrm{AA}$ at the electrode. All the 
optimum values were chosen by compromising the peak height, stability of the base line, no positive blank signals, low analysis time, availability and economy, respectively. The optimised parameters range studied and the optimum values are listed in Table I.
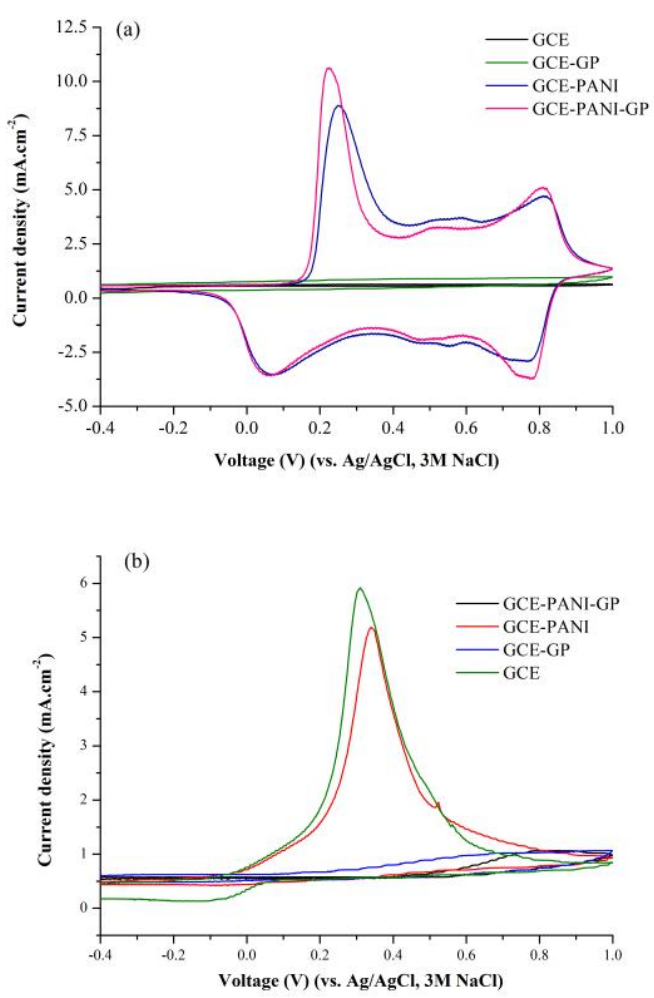

Fig. 3. CVs of bare GCE and GCEs modified with different prepared nanomaterials in (a) $1.0 \mathrm{M} \mathrm{HCl}$ and (b) in $1.0 \mathrm{mM} \mathrm{AA}$ containing $0.1 \mathrm{M}$ phosphate buffer $(\mathrm{pH} 7)$ at a scan rate of $100 \mathrm{mV} \cdot \mathrm{s}^{-1}$.

TABLE I: OPTIMISED OPERATING CONDITIONS FOR THE DETERMINATION OF AA BY USE OF A GP-PANI MODIFIED GCE IN AN FI-AMPEROMETRIC

\begin{tabular}{lcc}
\multicolumn{3}{c}{ MANIFOLD } \\
\hline \multicolumn{1}{c}{ Parameter study } & Range studied & Optimum \\
\hline Concentration of phosphate buffer & $0.01-1.0$ & 0.1 \\
solution $(\mathrm{M})$ & & \\
pH of phosphate buffer & $4-9$ & 7 \\
Sample volume $(\mu \mathrm{L})$ & $25-150$ & 100 \\
flow rate $\left(\mathrm{mL} \cdot \mathrm{min}^{-1}\right)$ & $0.5-3.0$ & 1.5 \\
\hline
\end{tabular}

By using the proposed FI manifold (Fig. 1), the linearity of the relationship between the current signals versus varying AA concentrations (Fig. 4) was studied by injection of standard solutions into FI-amperometric system under the optimal conditions (Table I). The linear range of the calibration graph (a plot of the peak heights against the standard AA concentrations) was observed for the AA standards over the concentration range of $0.05-0.50 \mathrm{mM}\left(\mathrm{R}^{2}\right.$ $=0.9967$ ) with detection limit of $0.027 \mathrm{mM}$ and quantitation limit of $0.089 \mathrm{mM}$. The sensitivity of the method obtained was $4.5754 \mathrm{~mA} \cdot \mathrm{mM}^{-1}$. As shown in Table II, the data obtained by the proposed method are compared with the values obtained from normal phase HPLC (NP-HPLC) method. AA contents were expressed as mg AA per 100 gram of each sample. Using the paired t-test [15], the results obtained from the electrochemical FIA are not significantly different from the results of the chromatographic method. This suggests the potential use of the measurement system is optimal for determining AA in the real samples.

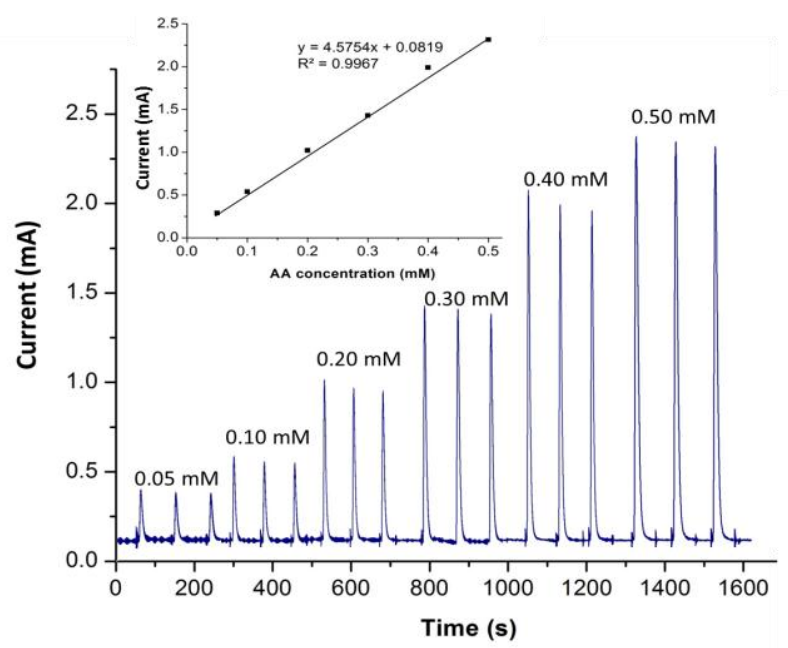

Fig. 4. FIAgram and calibration curve (inset) of AA oxidation at the GP-PANI-modified GCE at different concentrations in $0.1 \mathrm{M}$ phosphate buffer ( $\mathrm{pH} 7)$ at operated potential of $300 \mathrm{mV}$.

TABLE II: COMPARISON OF ASCORBIC ACID OF LONGANS AND LYCHEES OBTAINED BY USING THE PROPOSED FIA AND THE HPLC METHODS

\begin{tabular}{ccccc}
\hline \multirow{2}{*}{ Sample } & \multirow{2}{*}{ Cultivar } & \multicolumn{2}{c}{ AA (mg/100 g)* } & \multirow{2}{*}{ t-cal } \\
\cline { 3 - 4 } & & FIA & HPLC & \\
\hline \multirow{2}{*}{ Longan } & Daw & $64.17 \pm 1.42$ & $66.08 \pm 0.67$ & -1.896 \\
& Chompoo & $52.82 \pm 1.89$ & $53.21 \pm 0.64$ & -0.291 \\
& Haew & $57.33 \pm 0.91$ & $56.85 \pm 1.15$ & 0.746 \\
Lychee & Hong-Huay & $28.61 \pm 0.65$ & $30.11 \pm 1.28$ & -3.264 \\
& O-hia & $36.04 \pm 0.99$ & $36.83 \pm 0.82$ & -1.255 \\
& Chakrapad & $23.15 \pm 1.02$ & $24.79 \pm 0.45$ & -2.274 \\
\hline
\end{tabular}

${ }^{*}$ average of triplicate results, $\mathrm{t}$-cal represent calculated t-value experimentally $\mathrm{t}$ from the t-table at $95 \%$ confident level, 2 degree of freedom is 4.30

\section{CONCLUSIONS}

GP-PANI nanostructure electrode has been successfully employed in the electrochemical FIA system for the AA assay in fruits. Results obtained by the developed method showed good agreement with those obtained by NP-HPLC technique verified by the student's t-test at 95\% confident level (table II), which presented the good sensitivity, reproducibility which would be suitable for analysing a wide range of antioxidants (reducing agents) in several sample matrices.

\section{ACKNOWLEDGEMENTS}

The financial support from the Research, Development and Engineering (RD\&E) fund through The National Nanotechnology Center (NANOTEC), The National Science and Technology Development Agency (NSTDA), Thailand (P-11-00992) to Chiang Mai University (CMU), the Center of Excellence for Innovation in Chemistry (PERCH-CIC), the Institute for the Promotion of Teaching Science and Technology (IPST), the Thailand Research Fund (MRG5680058), Graduate School (CMU) and an award of CMU Young Researcher 2012 (K.O.) is deeply acknowledged. Debts are also due to the research funds from National Research Council of Thailand, (NRT 2V research Program 2013), The National Research University (NRU) Project under Thailand's Office of the commission on Higher Education (CHE), and Material Science Research Center 
CMU for their very kind partial supports. Special thanks are sincerely expressed to Science and Technology Institute for granting an excellent electrochemical detector to Alphaflow Research Laboratory, Faculty of Science, Chiang Mai University, Thailand.

\section{REFERENCES}

[1] J. M Zen, D. M. Tsai, and A. S. Kumar, "Flow injection analysis of ascorbic acid in real samples using a highly stable chemically modified screen-printed electrode," Electroanal., vol. 15, pp. 1171-1176, 2003.

[2] N. E. Llamas, M. S. D. Nezio, and B. S. F. Band, "Flow-injection spectrophotometric method with on-line photodegradation for determination of ascorbic acid and total sugars in fruit juices," J. Food Compos. Anal., vol. 24, pp. 127-130, 2011.

[3] S. Yilmaz, M. Sadikoglu, G. Saglikoglu, S. Yagmur, and G. Askin, "Determination of ascorbic acid in tablet dosage forms and some fruit juices by DPV," Int. J. Electrochem. Sci., vol. 3, pp. 1534-1542, 2008.

[4] A. R. B. de Quiros, M. F. Arias, and J. L. Hernandez, "A screening method for the determination of ascorbic acid in fruit juices and soft drinks," Food Chem. vol. 116, pp. 509-512, 2009.

[5] S. P. Arya, M. Mahajan, and P. Jain, "Non-spectrophotometric methods for the determination of Vitamin C," Anal. Chim. Acta, vol 417, pp.1-14, 2000.

[6] A. V. Pereira and O. Fatibelo-Filho, "Spectrophotometric flow injection determination of L-ascorbic acid with a packed reactor containing ferric hydroxide," Talanta, vol. 47, pp. 11-18, 1998.

[7] N. Lenghor, J. Jakmunee, M. Vilen, R. Sara, G. D. Christian, and K. Grudpan, "Sequential injection redox or acid-base titration for determination of ascorbic acid or acetic acid," Talanta, vol. 58, pp. 1139-1144, 2002.

[8] L. Ozcan, M. Sahin, and Y. Sahin, "Electrochemical preparation of a molecularly imprinted polypyrrole-modified pencil graphite electrode for determination of ascorbic acid," Sensors, vol. 8, pp. 5792-5805, 2008.

[9] A. K. Roy, V. S. Nisha, C. Dhand, and B. D. Malhotra, "Molecular imprinted polyaniline film for ascorbic acid detection," J. Mol. Recogn. vol. 24, pp. 700-706, 2011.
[10] A. Ambrosi, A. Morrin, M. R. Smyth, and A. J. Killard, "The application of conducting polymer nanoparticle electrodes to the sensing of ascorbic acid," Anal. Chim. Acta, vol. 609, pp. 37-43, 2008

[11] S. Pakapongpan, J. P. Mensing, D. Phokharatkul, T. Lomas, and A. Tuantranont, "Highly selective electrochemical sensor for ascorbic acid based on a novel hybrid graphene-copper phthalocyanine-polyaniline nanocomposites," Electrochim. Acta, vol 133, pp. 294-301, 2014

[12] J. Luo, S. Jiang, R. Liu, Y. Zhang, and X. Liu, "Synthesis of water dispersible polyaniline/poly(styrenesulfonic acid) modified graphene composite and its electrochemical properties," Electrochim. Acta, vol. 96, pp. 103-109, 2013.

[13] L. D. Leong and G. Shui, "An investigation of antioxidant capacity of fruits in Singapore markets," Food. Chem., vol. 76, pp. 69-75, 2002.

[14] S. B. Yoon, E. H. Yoon, and K. B. Kim, "Electrochemical properties of leucoemeraldine, emeraldine, and pernigraniline forms of polyaniline/multi-wall carbon nanotube nanocomposites for supercapacitor applications," J. Power Sources., vol. 196, pp. 10791-10797, 2011.

[15] C. Miller and J. N. Millers, Statistics for analytical chemistry, 3rd ed. New York: Ellis Horwood PTR Prentice Hall, 1993.

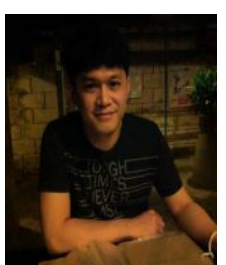

Kontad Ounnunkad received his Ph.D. in chemistry from University of Wollongong, Australia since 2011. He is appointed as a lecturer in Department of Chemistry, Faculty of Science, Chiang Mai University, Chiang Mai, Thailand. His research interests are fabrications of the nanomaterials, conducting polymers, electrochemical sensors and electrochemical devices.

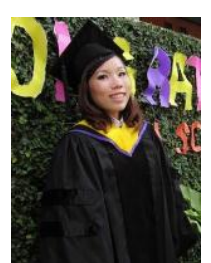

Poachanee Norfun is a Ph.D. student in chemistry, Department of Chemistry, Faculty of Science, Chiang Mai University, Thailand. She got her M.Sc. degree in chemistry in 2009 from Chiang Mai University, Chiang Mai, Thailand. Her current research interests include flow injection technique based on electrochemical detection, nanomaterial and electrochemical sensor. 